\title{
Deep Breathing Governs Binocular Rivalry
}

\author{
Ahmad Yousef ${ }^{1}$ \\ ${ }^{1}$ School of Computational Science and Engineering, McMaster University, Hamilton, Ontario, Canada \\ *Correspondence: mohamas2@mcmaster.ca
}

\begin{abstract}
Scientists had shown that visual rivalries, whether it's monocular or binocular have highly correlated temporal patterns of oscillations, see reference 1. Inhalation and exhalation, however, can be cognitively controlled, and these neurophysiological top-down processes can perfectly and timely alter the bistable visual motion perception, see reference 2 . In this study, therefore, we decide to investigate whether deep breathing can also control the binocular rivalry. Astoundingly, we found that deep breathing is the best governor of binocular rivalry, it has the ability to significantly elongate the dominance duration. Moreover, the human subjects have sufficient control over their visually aware; namely, they usually see a stimulus during deep inhalation \& the other stimulus during deep exhalation. Introduction
\end{abstract}

Binocular rivalry, a bistable visual perception in which human visual awareness alternates between different visual stimuli presented to each eye, is an astounding neurophysical approach to quantify consciousness. Interesting to

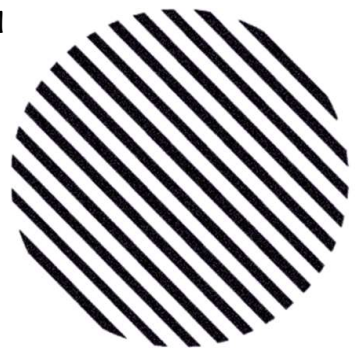
mention, both kinds of attention, involuntary or voluntary, assist in increasing the visual consciousness dominance duration of the corresponding stimulus. In another word, attention controls the human visual awareness to some extent.

Strength (Levelt, 1965), saliency (Engle, 1956), spiral motion (Malek, 2012), higher spatial frequency (Fahle, 1982), duchenne expressions (Malek, 2018), are all having 'attractive' visually stimulating features, namely, they all trigger automatic attention; therefore they all dominate the visual awareness over 'non-attractive' ones. Voluntary attention to certain details to a certain stimulus also elongates the awareness dominance duration of the stimulus (Lake, 1978).

All of the aforementioned parameters, however, were not able to control the rivalry thoroughly, namely, there is some control to increase/decrease the visual awareness dominance duration, but none of these parameters can be considered as a governing controller (an actual switcher) for binocular rivalry. Given that the deep breathing governs the switches of visual motion bistable perception, see reference 2 , and since different kinds of perceptual rivalries produce highly correlated temporal patterns of oscillations, see reference 1; we were motivated to test the influence of deep breathing on binocular rivalry setting.

Astonishingly, we found that deep breathing is the best controller to switch the dominant percept in binocular rivalry, namely, the human subjects were generally visually aware about certain stimulus with deep inhalation, and they were aware about other stimulus with deep exhalation. We presently may emphasize, the perceptual switcher might reside outside the visual cortex, but rather it might reside in the primary olfactory cortex. Since deep breathing achieves

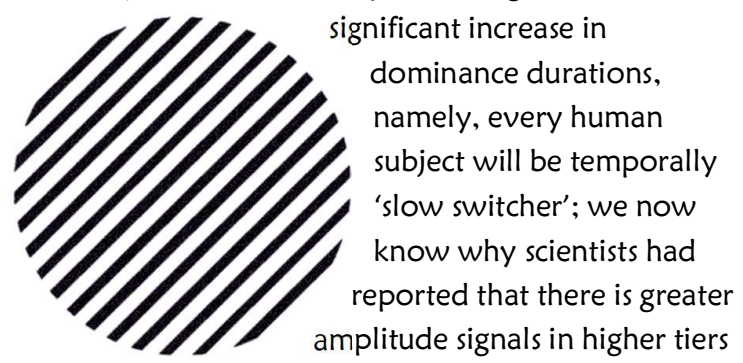

of ventral pathway associated with slow switchers, see reference 9. It might be because the primary olfactory cortex is a close neighbor to the aforementioned areas. The control signal however might start at the dorsolateral prefrontal cortex, a signal that may feed both of the visual cortex, and the primary olfactory cortex.

\section{Materials \& Methods}

Twelve human subjects, with average pupils, were recruited to participate in this study. Each circle in each stimulus subtends exactly 8.76 DVA, namely, 3.7 centimeters, on the screen, whereas the human subjects are 24 centimeters away from the screen, with 9.25 diopters base-out glasses, see reference 10. Important to mention, we had used the stimulus at the center of this page, and then flipped it 90 degrees for counterbalancing purposes. The experiment has the two trials, normal trial (two minutes of rivalry without deep breathing versus two minutes of rivalry with deep 
breathing), \& the aforementioned steps were repeated for the counterbalancing trial. The stimuli are built with tilted lines $\left(45^{\circ}\right)$ for the right-side stimulus versus tilted lines $\left(135^{\circ}\right)$ for the right-side; and ,the counterbalancing trial is the same stimulus but flipped $180^{\circ}$. Each human subject has to undergo into the experiment for three times. Technically speaking, there might be some human subjects suffering from amblyopia or breathing problems; and therefore, they might not be able to experience neither the binocular rivalry nor how deep breathing influences binocular rivalry. In our experiment, luckily, all of the subjects were able to experience binocular rivalry, as well as, how deep breathing affects it from the first trial. For quantitative analysis, whether the human subject in the normal session, or deep breathing session; with a specialized timer, each subject has to record every switch, key press 1 for right-sided stimulus, and key press 2 for the left-sided stimulus. The behavioral data out of all trials are then averaged for each human subject; then the 'twelve' mean values afterwards are used to estimate the mean value, and standard error of the mean. To comply to Helsinki declaration, however, we confirmed comfortable settings for each human subject. Eventually, at the end of each trial, we have to confirm with each human subject whether he/she is able to have full control to switch the rivalry through deep breathing. Expect one of the subjects who had reported experience closer to 'ultimate' control, their answers were truly astonishing; all of the human subjects have reported 'ultimate' control over the rivalry. Interesting to mentioned, deep breathing does not only control the binocular rivalry, but it significantly elongates the dominance duration, as shown in the up-central figure. In a separate session, one of the subjects had 'reperformed' the experiment in the very morning without breakfast, and she claimed that she is presently in meditative state, the subject had noticed that she is able to stop the rivalry indefinitely with a plaid visual stimulus, namely, she might be in a state closer to what's offered in reference 12 . We therefore encourage scholars who had access to people performing mediation to confirm the effect of deep breathing on them, thus universality of the aforementioned observation.

Results and Discussion In addition to its outstanding control over the binocular rivalry; as shown in the up-central infographic, deep breathing had a great ability to significantly elongates the dominance duration; deep inhalation lasts more few seconds than deep exhalation though. Human subjects had reported that the binocular rivalry get back to normal at the end of exhalation process. Important to mention, they had also reported no mixed percepts during deep breathing stages, unlike their experience with rivalry without deep breathing. It is important to mention that GABA concentration has a positive correlation with the dominance duration of binocular rivalry stimuli (van Loon et al., 2013); as if deep breathing alters the activation of GABA machinery.

\section{Essential Notification}

Stimuli and the BR setup can be found in reference 10 . Readers who don't have binocular rivalry setup are welcome to visit our lab. The laboratory has binocular rivalry glasses that's compatible with every brain imaging machines, because it doesn't have any attached metals; and we wish to collaborate with scholars with brain imaging facilities, especially MEC. EEG, MRI; in addition to specialized eye tracker, and ECC facilities. Enquiries should be sent to the author.

Transactional References

[1] Carter, Pettigrew, 2003. A common oscillator for perceptual rivalries?. Perception.

[2] Yousef, A. 2019. “Deep Breathing Alters Visual Motion Perception.” PsyArXiv. doi:10.31234/osf.io/up3sa.

[3] Levelt (1965). Binocular brightness averaging and contour information. British Journal of Psychology.

[4] Engle (1956). The role of content in binocular resolution. The American Journal of Psychology.

[5] Malek, etal. (2012). Binocular rivalry of spiral and linear moving random dot patterns in human observers.

[6] Fahle (1982). Binocular rivalry: suppression depends on orientation and spatial frequency. Vision Research.

[7] Malek, (2018). Generalizing Duchenne to sad expressions with binocular rivalry and perception ratings. Emotion.

[8] Lack (1978). Selective attention and the control over binocular rivalry, Dissertation.

[9] - (2019). Tagged MEG measures binocular rivalry in a cortical network that predicts alternation rate. POLSONE.

[10]Stimuli and the BR system may be found here: https://drive.google.com/drive/folders/1UxGlA-

6LEr3kQKSBQGsEqSrj4F5t_2J6

[11] van Loon et al., (2013). GABA shapes the dynamics of bistable perception. Current Biology.

[12] Olivia Carter, etal. (2005). Meditation alters perceptual rivalry in Tibetan Buddhist monks. Current Biology. 